\title{
ERRATUM
}

\section{Erratum to: Advances in Therapy}

(c) Springer Healthcare 2017

Erratum to: Adv Ther 2016 33(12)

Publisher's Erratum

Due to an internal error, the cover month of Volume 33 Issue 12 of Advances in Therapy was originally incorrectly labelled in the XML data as January 2017, instead of December 2016. This has now been updated. This update does not affect the articles published in Volume 33 Issue 12 , which were correctly dated and paginated. 